\title{
More to Movement Disorders Than Motion
}

\author{
By Jack M. Gorman, MD
}

Parkinson's disease has always been considered one of the neurological disorders for which the underlying pathology is precisely known-the progressive lost neurons in the pars compacta of the substantia nigra, a major structure in the basal ganglia. The disorder, first described in 1817 by James Parkinson, has characteristic clinical features, can be confirmed even in fairly early cases by positron emission tomography using the radiotracer fluorodopa 18 , and is relatively easy to characterize on postmortem exam with the loss of dopamine cells and presence of Lewy bodies. Replacement therapy with levodopa and dopamine agonists are among the strategies that offer relief to patients with Parkinson's disease.

Despite all of this knowledge, understanding and treating Parkinson's disease has frustrated neurologists and neuroscientists for decades. It is precisely because the underlying pathology seems so clear that scientists believed it should be much easier to discover causes and definitive treatment than was supposed to be the case for seemingly more etiologically obscure neuropsychiatric disorders, such as schizophrenia and migraine headache. Yet, despite decades of research funded by both the federal government and several active private philanthropies, we are still in the dark about what initiates the inexorable loss of dopamine neurons in the substantia nigra or how to offer most patients long-lasting relief. The brain, as usual, confounds us when we try to cure its diseases.

This issue of CNS Spectrums makes clear that Parkinson's disease is more than a tremor and that its diagnosis is not always straightforward. Other movement disorders can sometimes make differential diagnosis difficult, and there is a psychogenic version of the condition that is especially troublesome when trying to rule Parkinson's disease in or out. Patients with long-standing Parkinson's disease suffer from dementia, often characterized (many would say superficially) as a prototypical "sub-cortical dementia." Patients also suffer from a variety of psychiatric illnesses. Chronic use of many of the medications indicated to treat Parkinson's disease ultimately causes psychotic symptoms. When the typical antipsychotics, such as haloperidol, were all we had, clinicians treating psychosis in a Parkinson's disease patient were caught between the proverbial rock and a hard place. Antipsychotics are effective in relieving psychotic symptoms, but they often spur on a troublesome group of extrapyramidal side effects that prominently include parkinsonian syn- drome. However, neurologists have welcomed medications such as olanzapine, quetiapine, and clozapine, which are effective antipsychotics with little potential for extrapyramidal side effects.

Perhaps the most common psychiatric problem among Parkinson's disease patients is depression. This is probably partly caused by the trauma and stress of having a debilitating and chronic disease and partly from the effect of decreased dopamine neural transmission. One of my medical school classmates and closest friends, Susan Bressman, MD, is chief of neurology at New York City's Beth Israel Medical Center and an internationally recognized expert on movement disorders such as Parkinson's disease. She routinely refers patients to psychiatrists who must grapple with these issues, knowing that selective serotonin reuptake inhibitors sometimes exacerbate the tremor and other symptoms of Parkinson's disease.

Deep brain stimulation, in which an electrode is placed in regions of the brain (eg, the globus pallidum and the subthalamic nucleus of the thalamus) is an intriguing and exciting experimental approach to treating Parkinson's disease and depression. Its exact mechanism of action is unclear, but understanding its mechanism of action and refining the procedure is likely to be a subject of intense research interest over the next few years.

We know that it is simplistic to state diabetes mellitus is simply a disease of insulin deficiency. Patients with diabetes suffer from more than just metabolic problems, facing conditions such as kidney and vascular disease that are only indirectly attributable to the loss of normal insulin levels. Similarly, although James Parkinson thought he was describing a disease mainly characterized by "palsy," it is clear that Parkinson's disease involves protean alteration to brain function resulting in a host of neuropsychiatric symptoms. We have discovered that treating Parkinson's disease requires the coordinated efforts of both psychiatrists and neurologists, and sometimes even neuroradiologists and neurosurgeons. Thus, Parkinson's disease is one of the most fitting topics imaginable for CNS Spectrums, as this journal seeks to join psychiatrists and neurologists in an effort to better understand and combat brain disease. As we continue in this effort, we are extremely grateful for the vision and insight of this month's guest editor, Joseph Jankovic, MD, and the authors of this excellent collection of articles. CNS

Dr. Gorman is the editor of this journal and adjunct professor of psychiatry at Mount Sinai School of Medicine in New York City. 